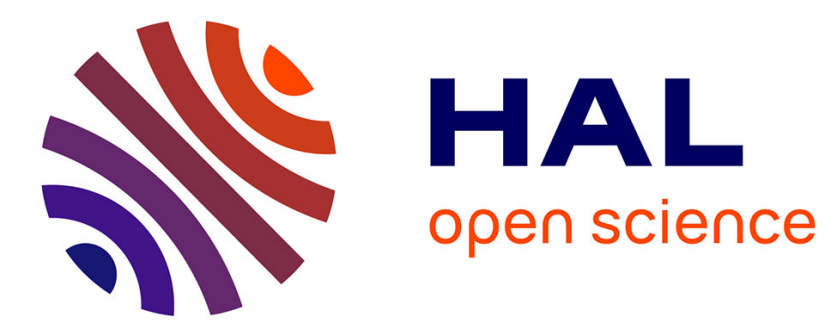

\title{
State estimation of nonlinear systems using multiple model approach
}

\author{
Dalil Ichalal, Benoît Marx, José Ragot, Didier Maquin
}

\section{To cite this version:}

Dalil Ichalal, Benoît Marx, José Ragot, Didier Maquin. State estimation of nonlinear systems using multiple model approach. American Control Conference, ACC 2009, Jun 2009, St Louis, Missouri, United States. pp.4636-4641, 10.1109/ACC.2009.5160393 . hal-00355833

\section{HAL Id: hal-00355833 https://hal.science/hal-00355833}

Submitted on 10 Apr 2014

HAL is a multi-disciplinary open access archive for the deposit and dissemination of scientific research documents, whether they are published or not. The documents may come from teaching and research institutions in France or abroad, or from public or private research centers.
L'archive ouverte pluridisciplinaire HAL, est destinée au dépôt et à la diffusion de documents scientifiques de niveau recherche, publiés ou non, émanant des établissements d'enseignement et de recherche français ou étrangers, des laboratoires publics ou privés. 


\title{
State estimation of nonlinear systems using multiple model approach
}

\author{
Dalil Ichalal, Benoît Marx, José Ragot and Didier Maquin
}

\begin{abstract}
This paper addresses the problem of state estimation of nonlinear systems described by a Takagi-Sugeno multiple model with unmeasurable decision variables. The method is based on the reformulation of the multiple model in an equivalent form. First, the convergence conditions of the state estimation error are established using the Lyapunov method and they are expressed in LMI formulation. Secondly, performances of the observer are enhanced by pole clustering and $\mathcal{L}_{2}$ attenuation of bounded exogenous disturbances. Finally, the method is applied to estimate the state of a link flexible joint robot.
\end{abstract}

\section{INTRODUCTION}

The problem of designing observers for nonlinear systems constitutes an important field of research these last years. It is due to a large domain of application such as, estimating of the not accessible states, when sensors are not available or expensive, designing feedback control laws and generating residual signals in order to detect and isolate failures.

Early work on the state estimation of nonlinear systems dates back to 1973 when Thau [19] proposed an extension of the Luenberger observer [11] to Lipschitz systems, he then provided sufficient conditions for the convergence of the state estimation error, however, he did not propose a methodology for synthesizing the observer gain. In [15], authors have used the same structure of observer but they provided an iterative method for obtaining the observer gain. Unfortunately, Raghavan's algorithm often fails to obtain a solution even if the observability condition is satisfied and it fails if the Lipschitz constant has a large value. Raghavan then proposed a state transformation to relax the design conditions. In [16] Rajamani gave a solution to these problems. He obtained necessary and sufficient conditions on the observer matrix that ensure asymptotic stability of the observer and proposed a design procedure, based on the use of a gradient based optimization method. He discussed the equivalence between the stability condition and the $H_{\infty}$ minimization in the standard form, and pointed out that this design method was not solvable since the regularity assumptions are not satisfied. In recent work [14], based on the result of Rajamani [13], Pertew proposed a dynamic observer. The problem of regularity assumptions pointed out in [16] is solved by modifying the $H_{\infty}$ problem. Other classes of nonlinear systems are also studied in the literature to design observers for nonlinear systems, among them, we can cite the use of Linear Parameter Varying systems (LPV) [3].

All the authors are with the Centre de Recherche en Automatique de Nancy (CRAN), Nancy-Université, 2, avenue de la forêt de Haye 54516 Vandoeuvre-les-Nancy \{dalil.ichalal, benoit.marx, jose.ragot, didier.maquin\}eensem.inpl-nancy.fr
In this paper another method is proposed by using a Takagi-Sugeno multiple model approach to design observers for nonlinear systems. We consider the case where the weighting functions depend on unmeasurable decision variables (the state of the system). Section II introduces the problem and some background. In Section III, the main results to design the observer are given under LMI formulation. After, the result to minimization of the $\mathcal{L}_{2}$ norm of the transfer from bounded unknown exogenous disturbances to the state estimation error is proposed. The performances of the observer are improved by eigenvalues assignment. Finally, in section IV, a simulation example is given.

\section{TAKAGI-SUGENO MULTIPLE MODEL APPROACH}

Let us consider the multiple model representation of a nonlinear system:

$$
\left\{\begin{array}{l}
\dot{x}(t)=\sum_{i=1}^{r} \mu_{i}(\xi(t))\left(A_{i} x(t)+B_{i} u(t)\right) \\
y(t)=C x(t)
\end{array}\right.
$$

$x(t) \in \mathbb{R}^{n}$ is the state vector, $u(t) \in \mathbb{R}^{m}$ is the control input and $y(t) \in \mathbb{R}^{p}$ is the measurement output. $A_{i}, B_{i}$ and $C$ are real known matrices with appropriate dimensions. The weighting functions $\mu_{i}$ are nonlinear in $\xi(t)$ and satisfy the convexity property:

$$
\left\{\begin{array}{l}
\sum_{i=1}^{r} \mu_{i}(\xi(t))=1 \\
0 \leq \mu_{i}(\xi(t)) \leq 1, i \in\{1, \ldots, r\}
\end{array}\right.
$$

The decision variable $\xi(t)$ can depend on measurable signals, for example the input $u(t)$ or the output $y(t)$ of the system (this situation is largely studied in the literature), or on unmeasurable signals like the state $x(t)$. The next section gives a comparison between using measurable or unmeasurable signals.

\section{A. Motivations}

Takagi-Sugeno multiple model has proved its effectiveness in the study of nonlinear systems. Indeed, it gives a simpler formulation from the mathematical point of view to represent the behavior of nonlinear systems [17]. In the field of stability analysis and stabilization, many works, such as state feedback control [18][10][7], have been developed and applied in a lot of practical situations. The problem of state estimation has also been studied in order to design state feedback control laws and design a residual generator to detect and isolate faults in the system and reconfiguring the control laws in the presence of faults [2][5]. 
In the field of state estimation and diagnosis of nonlinear systems using multiple model approach, the most of the published works considered T-S models with measurable premise variables [2][13]. It is clear that the choice of measurable premise variables offers a good simplicity to generalize the methods already developed for linear systems. But in the case where the premise variables are not measurable, the problem becomes very hard. However, this formalism is very important in both the exact representation of the nonlinear behavior by multiple model (see the simulation example) and in diagnosis method based on observer banks to detect and isolate actuator and sensor faults. Indeed in this case, the use of measurable premise variables requires to develop two different multiple models, the first using the input $u(t)$ in the premise variable to detect and isolate sensor faults, and the second using the output of the system for actuator faults. Multiple model with unmeasurable premise variables allows to develop only one model of the system behavior to detect and isolate actuator and sensor faults using observer banks. In the literature, a few works are devoted to the case of unmeasurable decision variables, nevertheless, we can cite [5][12][4], where the authors proposed the fuzzy ThauLuenberger observer which is an extension of the classical Luenberger observer. The main contributions of this paper are to reduce the conservatism of the existing works [4][5] by reducing the number of LMIs to be solved and relaxing the conditions under which the method is applicable.

\section{MAIN RESULTS}

Consider the following nonlinear system described by a multiple model:

$$
\left\{\begin{array}{l}
\dot{x}(t)=\sum_{i=1}^{r} \mu_{i}(x(t))\left(A_{i} x(t)+B_{i} u(t)\right) \\
y(t)=C x(t)
\end{array}\right.
$$

Let us consider the matrices $A_{0}$ and $\bar{A}_{i}$ defined in one of the two following ways:

1) The matrix $A_{0}$ is defined as a mean of the matrices $A_{i}$ then:

$$
A_{0}=\frac{1}{r} \sum_{i=1}^{r} A_{i}
$$

2) The matrix $A_{0}$ is chosen as a dominant local model of the system. Let the $j^{\text {th }}$ local model is the dominant one, then:

$$
A_{0}=A_{j}
$$

The matrix $\bar{A}_{i}$ is then defined by:

$$
\bar{A}_{i}=A_{i}-A_{0}
$$

By substituting $A_{0}$ and $\bar{A}_{i}$ in the state equation (3), we obtain the following equivalent system:

$$
\dot{x}(t)=A_{0} x(t)+\sum_{i=1}^{r} \mu_{i}(x(t))\left(\bar{A}_{i} x(t)+B_{i} u(t)\right)
$$

The proposed observer is in the following form:

$$
\begin{aligned}
& \dot{\hat{x}}(t)=A_{0} \hat{x}(t)+\sum_{i=1}^{r} \mu_{i}(\hat{x}(t))\left(\bar{A}_{i} \hat{x}(t)+B_{i} u(t)\right)+L(y(t)-\hat{y}(t)) \\
& \hat{y}(t)=C \hat{x}(t)
\end{aligned}
$$

The state estimation error is given by:

$$
e(t)=x(t)-\hat{x}(t)
$$

and its dynamic is:

$$
\dot{e}(t)=\left(A_{0}-L C\right) e(t)+\Delta(x, \hat{x}, u)
$$

where:

$$
\begin{aligned}
\Delta(x, \hat{x}, u) & =\sum_{i=1}^{r}\left[\bar{A}_{i}\left(\mu_{i}(x(t)) x(t)-\mu_{i}(\hat{x}(t)) \hat{x}(t)\right)\right. \\
& \left.+B_{i}\left(\mu_{i}(x(t))-\mu_{i}(\hat{x}(t)) u(t)\right)\right]
\end{aligned}
$$

Assumption 1: Assume that the following conditions holds:

- A1. $\left|\mu_{i}(x(t)) x(t)-\mu_{i}(\hat{x}(t)) \hat{x}(t)\right|<\alpha_{i}|x(t)-\hat{x}(t)|$

- A2. $\left|B_{i}\left(\mu_{i}(x(t))-\mu_{i}(\hat{x}(t))\right)\right|<\beta_{i}|x(t)-\hat{x}(t)|$

- A3. $|u(t)|<\rho$

where $\alpha_{i}>0$ and $\beta_{i}>0$.

The calculation of $\alpha_{i}$ and $\beta_{i}$ is given by the following steps: Consider a function $f(x): x \in R^{n} \rightarrow R^{n}$ defined as follows:

$$
f(x)=\left[\begin{array}{lll}
f_{1}(x)^{T} & \cdots & f_{n}(x)^{T}
\end{array}\right]^{T}, x=\left[\begin{array}{lll}
x_{1}^{T} & \cdots & x_{n}^{T}
\end{array}\right]^{T}
$$

The Taylor formula at order zero with an integral remainder term of $f(x)$ around $\hat{x}$ is:

$$
f_{i}(x)-f_{i}(\hat{x})=\int_{\hat{x}_{1}}^{x_{1}} \frac{\partial f_{i}}{\partial x_{1}}(t) d t+\ldots+\int_{\hat{x}_{n}}^{x_{n}} \frac{\partial f_{i}}{\partial x_{n}}(t) d t
$$

$i \in\{1, \ldots, n\}$. Each function $f_{i}$ can be bounded as follows:

$$
\left|f_{i}(x)-f_{i}(\hat{x})\right| \leq \int_{\hat{x}_{1}}^{x_{1}}\left|\frac{\partial f_{1}}{\partial x_{1}}(t)\right| d t+\ldots+\int_{\hat{x}_{n}}^{x_{n}}\left|\frac{\partial f_{1}}{\partial x_{n}}(t)\right| d t
$$

Let define $\left.a_{i j}=\max _{t \in\left[x_{j}\right.} \hat{x}_{j}\right]\left|\frac{\partial f_{i}}{\partial x_{j}}(t)\right| i, j \in\{1, . ., n\}$. The interval $\left[\begin{array}{ll}x_{j} & \hat{x}_{j}\end{array}\right]$ is not known, so $a_{i j}$ is calculated for $t \in R$. We obtain $a_{i j}=\max _{t \in R}\left|\frac{\partial f_{i}}{\partial x_{j}}(t)\right|$. Then, (13) can be re-written as follows:

$$
\left|f_{i}(x)-f_{i}(\hat{x})\right| \leq a_{i 1}\left|x_{1}-\hat{x}_{1}\right|+\ldots+a_{i n}\left|x_{n}-\hat{x}_{n}\right|
$$


By rewriting the above inequalities in matrix form, we obtain:

$$
|f(x)-f(\hat{x})| \leq J|x-\hat{x}|
$$

where:

$$
J=\left[\begin{array}{ccc}
a_{11} & \cdots & a_{1 n} \\
\vdots & \ddots & \vdots \\
a_{n 1} & \cdots & a_{n n}
\end{array}\right]
$$

The Lipschitz constant of $f(x)$ is given by the maximum singular value of $J$.

Remark 1: The weighting functions $\mu_{i}(x)$ are globally Lipschitz. In the assumption A1,

- if the functions $\mu_{i}(x) x$ are globally Lipschitz then even if the system is unstable the constants $\alpha_{i}$ exist and the proposed method can be applied.

- If the functions $\mu_{i}(x) x$ are locally Lipschitz the stability of the system is required.

Note that the method proposed in [5] requires the stability of the system.

Using the assumptions A1, $\mathbf{A} 2$ and $\mathbf{A} 3$ the term $\Delta(x, \hat{x}, u)$ can be bounded as follows:

$$
|\Delta(x, \hat{x}, u)|<\gamma|x(t)-\hat{x}(t)|
$$

where:

$$
\gamma=\sum_{i=1}^{r}\left(\bar{\sigma}\left(\bar{A}_{i}\right) \alpha_{i}+\beta_{i} \rho\right)
$$

where $\bar{\sigma}(M)$ represents the maximum singular value of the matrix $M$.

Theorem 1: The state estimation error between the multiple model (7) and the multiple observer (8) converges asymptotically toward zero, if there exists matrices $P=P^{T}>$ 0 and $Q=Q^{T}>0$ and gain matrix $K$ such that the following condition holds:

$$
\left[\begin{array}{cc}
A_{0}^{T} P+P A_{0}-C^{T} K^{T}-K C+\gamma^{2} Q & P \\
P & -Q
\end{array}\right]<0
$$

The gain of the observer is computed by $L=P^{-1} K$.

Proof: The convergence condition of the state estimation error is obtained by using a quadratic Lyapunov function:

$$
V(t)=e(t)^{T} P e(t), \quad P=P^{T}>0
$$

its derivative is given by:

$$
\dot{V}(t)=\dot{e}(t)^{T} P e(t)+e(t)^{T} P \dot{e}(t)
$$

By substituting (10) in (18) we obtain:

$$
\dot{V}(t)=e(t)^{T}\left(\Phi^{T} P+P \Phi\right) e(t)+2 e(t)^{T} P \Delta(x, \hat{x}, u)
$$

where $\Phi=A_{0}-L C$.
Lemma 1: For two matrices $X$ and $Y$ with appropriate dimensions, the following property holds:

$$
X^{T} Y+X Y^{T}<X^{T} \Omega^{-1} X+Y \Omega Y^{T}, \quad \Omega>0
$$

For $Q>0$, by using lemma 1 and assumptions 1 in (19), we obtain:

$$
\begin{array}{r}
e(t)^{T}\left(\Phi^{T} P+P \Phi+P Q^{-1} P\right) e(t) \\
+\Delta(x, \hat{x}, u)^{T} Q \Delta(x, \hat{x}, u)<0
\end{array}
$$

Taking into account (14), the negativity of $\dot{V}(t)$ is assured if:

$$
e(t)^{T}\left(\Phi^{T} P+P \Phi+P Q^{-1} P+\gamma^{2} Q\right) e(t)<0
$$

The inequality (21) holds if:

$$
\left(A_{0}-L C\right)^{T} P+P\left(A_{0}-L C\right)+P Q^{-1} P+\gamma^{2} Q<0
$$

The condition (22) is not linear with respect to the variables $P, L$ and $Q$. In order to solve them with the classical LMI approaches, the change of variable $K=P L$, and the Schur complement [6] are used. The condition given in theorem 1 is obtained.

Example 1: Consider the following example to show the advantages of using the proposed design procedure. This is an example of a second-order system with:

$$
\begin{gathered}
A=\left[\begin{array}{ll}
3 & -1 \\
0 & -2
\end{array}\right], B=\left[\begin{array}{l}
0 \\
1
\end{array}\right], f(x)=\left[\begin{array}{c}
k \sin \left(x_{1}\right) \\
0
\end{array}\right], \\
C=\left[\begin{array}{ll}
1 & 0
\end{array}\right]
\end{gathered}
$$

The Lipschitz constant is $|k|$. The maximum Lipschitz values for which there exit a solution is 2.2360 for the technique proposed in [19] and 2.1181 for that proposed in [1]. Using a nonlinear sector transformation approach (see [18]), we obtain two sub-models defined by:

$$
\begin{gathered}
A_{1}=\left[\begin{array}{cc}
3+k & -1 \\
0 & -2
\end{array}\right], A_{2}=\left[\begin{array}{cc}
3-0.2172 k & -1 \\
0 & -2
\end{array}\right], \\
B_{1}=B_{2}=B
\end{gathered}
$$

The weighting functions are are given by:

$$
\left\{\begin{array}{l}
\mu_{1}(z(t))=\frac{z(t)+0.2172}{1.2172} \\
\mu_{2}(z(t))=\frac{1-z(t)}{1.2172}
\end{array}\right.
$$

where $z(t)=\frac{\sin \left(x_{1}\right)}{x_{1}}$. Theorem 1 gives solutions for a greater value of the Lipschitz constant compared to those obtained above. The following table gives the admissible value of $\gamma$ defined in (15) with respect to different values of $k$.

\begin{tabular}{|l|l|l|l|}
\hline$k$ & 1 & 50 & 100 \\
\hline$\gamma$ & 165.02 & 138.59 & 106.79 \\
\hline
\end{tabular}


Note that the first component of the state vector $x(t)$ is unstable, then the method proposed in [4] for Takagi-Sugeno models with unmeasurable decision variables cannot be applied because the considered Lipschitz constant goes to $\infty$ which implies that there is no solution for LMIs proposed in [4].

As conclusion, the multiple model approach applied to the state estimation of Lipschitz nonlinear systems reduces the conservatism of the methods proposed in the literature with regard to the admissible Lipschitz constants. In addition, the number of LMIs to solve has been reduced. Indeed, in the method given in [4], the number of LMI depends on the number $r$ of sub-models which is not the case in the proposed approach.

\section{A. $\mathcal{L}_{2}$ attenuating observer}

In this section, the result proposed in the previous section is extended for nonlinear system affected by unknown exogenous disturbances $\omega(t) \in \mathcal{L}_{2}$. Our purpose is to design the observer gain $L$ such that the observer error dynamics are asymptotically stable and that the following specified $\mathcal{L}_{2}$ norm upper bound is simultaneously guaranteed.

$$
\frac{\|e(t)\|_{2}}{\|\omega(t)\|_{2}}<\xi, \xi>0
$$

The perturbed system is described by

$$
\left\{\begin{array}{l}
\dot{x}(t)=\sum_{i=1}^{r} \mu_{i}(x(t))\left(A_{i} x(t)+B_{i} u(t)+E_{i} \omega(t)\right) \\
y(t)=C x(t)
\end{array}\right.
$$

By using the matrices defined in (4), (5) and (6) we obtain the following equivalent system:

$$
\dot{x}(t)=A_{0} x(t)+\sum_{i=1}^{r} \mu_{i}(x(t))\left(\bar{A}_{i} x(t)+B_{i} u(t)+E_{i} \omega(t)\right)
$$

The observer is given by the equations (8).

Theorem 2: The optimally robust observer (8) for system (25) which satisfies (24), is determined by minimizing $\bar{\xi}$ under the following LMI constraints in the variables $P, K$ and $\bar{\xi}$ (real positive number):

$$
\left[\begin{array}{ccc}
\Theta & P & P E_{i} \\
P & -Q & 0 \\
E_{i}^{T} P & 0 & -\bar{\xi}_{I}
\end{array}\right]<0, i=1, \ldots, r
$$

where:

$$
\Theta=A_{0}^{T} P+P A_{0}-K^{T} P-P K+\gamma^{2} Q+I
$$

The gain of the observer is computed by $L=P^{-1} K$. The attenuation level is given by $\xi=\sqrt{\bar{\xi}}$.
Proof: The dynamic of the state estimation error between (26) and (8) is given by the following equation:

$$
\dot{e}(t)=\left(A_{0}-L C\right) e(t)+\Delta(x, \hat{x}, u)+\sum_{i=1}^{r} \mu_{i}(x(t)) E_{i} \omega(t)
$$

where $\Delta(x, \hat{x}, u)$ is defined in (11). Consider the following Lyapunov function:

$$
V(t)=e^{T}(t) P e(t), P=P^{T}>0
$$

then

$$
\begin{aligned}
\dot{V}(t) & =e(t)^{T}\left(\Phi^{T} P+P \Phi\right) e(t)+2 e(t)^{T} P \Delta(x, \hat{x}, u) \\
& +2 \sum_{i=1}^{r} \mu_{i}(x(t)) e(t)^{T} P E_{i} \omega(t)
\end{aligned}
$$

According to the above assumptions and the lemma 1 we have:

$$
\begin{aligned}
\dot{V}(t) & \leq e(t)^{T}\left(\Phi^{T} P+P \Phi+P Q^{-1} P+\gamma^{2} Q\right) e(t) \\
& +2 \sum_{i=1}^{r} \mu_{i}(x(t)) e(t)^{T} P E_{i} \omega(t)
\end{aligned}
$$

The condition which guarantees the boundedness of the $\mathcal{L}_{2}$ norm of the transfer from $\omega(t)$ to $e(t)$ (which satisfies (24)) is given by:

$$
\dot{V}(t)+e(t)^{T} e(t)-\xi^{2} \omega(t)^{T} \omega(t)<0
$$

Using the derivative of the Lyapunov function (32), we obtain:

$$
\begin{array}{r}
e(t)^{T}\left(\Phi^{T} P+P \Phi+P Q^{-1} P+\gamma^{2} Q+I\right) e(t) \\
+2 e(t)^{T} P \sum_{i=1}^{r} \mu_{i}(x(t)) E_{i} \omega(t)-\xi^{2} \omega(t)^{T} \omega(t)<0
\end{array}
$$

In matrix form we obtain:

$$
\left[\begin{array}{c}
e(t) \\
\omega(t)
\end{array}\right]^{T} \mathcal{M}\left[\begin{array}{c}
e(t) \\
\omega(t)
\end{array}\right]<0
$$

where :

$$
\mathcal{M}=\sum_{i=1}^{r} \mu_{i}(x(t))\left[\begin{array}{cc}
\Theta+P Q^{-1} P & P E_{i} \\
E_{i}^{T} P & -\xi^{2} I
\end{array}\right]
$$

Using, the change of variables $K=P L, \bar{\xi}=\xi^{2}$ and the Schur complement, we obtain the following LMI, which guarantees that (35) holds:

$$
\left[\begin{array}{ccc}
\Theta & P & P E_{i} \\
P & -Q & 0 \\
E_{i}^{T} P & 0 & -\bar{\xi}_{I}
\end{array}\right]<0, i=1, \ldots, r
$$




\section{B. Pole Assignment}

In order to increase the performances of the observer, for example, to ensure that the estimation error $e(t)$ of the observer have fast and well damped response, it is necessary to assign all eigenvalues of $\left(A_{0}-L C\right)$ in a specific region of the complex-plane. In order to ensure a minimal decay rate $a$ and a damping ratio, the eigenvalues of the system generating $e(t)$ are clustered in $S(a, R, q)$ defined by

$$
S(a, R, q)=\{z \in \mathbb{C}|| z+q \mid<R, \operatorname{Re}(z)<a, q>0, a>0\}
$$

(see [13][8]). The observer eigenvalue constraints can be verified by theorem 3 .

Theorem 3: The optimally robust observer (8) for system (25) which satisfies (24) and the eigenvalues of the matrix $\left(A_{0}-L C\right)$ have their eigenvalues in the region $S(a, R, q)$, is determined by minimizing $\bar{\xi}$ under the following LMI constraints in the variables $P, K$ and $\bar{\xi}$ (real positive number):

$$
\begin{aligned}
& {\left[\begin{array}{ccc}
\Xi & P & P E_{i} \\
P & -Q & 0 \\
E_{i}^{T} P & 0 & -\bar{\xi}_{I}
\end{array}\right]<0, i=1, \ldots, r} \\
& {\left[\begin{array}{cc}
-R P & q I+A_{0}^{T} P-C^{T} K^{T} \\
q I+P A_{0}-K C & -R P
\end{array}\right]<0}
\end{aligned}
$$

where :

$$
\Xi=A_{0}^{T} P+P A_{0}-K^{T} P-P K+\gamma^{2} Q+I+2 a P
$$

Proof: The proof is based on adding the constraints on the eigenvalues of the matrix $\left(A_{0}-L C\right)$. (see [13])

\section{Simulation Results}

Consider a one-link manipulator with revolute joints actuated by a DC motor [9] defined as follows: The equations of this system are given by:

$$
\left\{\begin{array}{l}
\dot{\theta}_{m}(t)=\omega_{m}(t) \\
\dot{\omega}_{m}(t)=\frac{k}{J_{m}}\left(\theta_{l}(t)-\theta_{m}(t)\right)-\frac{B}{J_{m}} \omega_{m}(t)+\frac{K_{\tau}}{J_{m}} u(t) \\
\dot{\theta}_{l}(t)=\omega_{l}(t) \\
\dot{\omega}_{l}(t)=-\frac{k}{J_{l}}\left(\theta_{l}(t)-\theta_{m}(t)\right)-\frac{m g h}{J_{l}} \sin \left(\theta_{l}(t)\right)
\end{array}\right.
$$

where $\theta_{m}(t)$ stands for the angular rotation of the motor, $\omega_{m}(t)$ is the angular velocity of the motor, $\theta_{l}(t)$ is the angular position of the link, and $\omega_{l}(t)$ is the angular velocity of the link. The input signal is given by $u(t)=\sin (t)$, and the initial condition are $x_{0}=0$ for the system and $\hat{x}_{0}=1$ for the observer. The state representation is:

$$
\dot{x}(t)=A x(t)+f(x(t))+B u(t)+E \omega(t), y(t)=C x(t)
$$

where:

$$
A=\left[\begin{array}{cccc}
0 & 1 & 0 & 0 \\
-48.6 & -1.25 & 48.6 & 0 \\
0 & 0 & 0 & 1 \\
1.95 & 0 & -1.95 & 0
\end{array}\right], B=\left[\begin{array}{c}
0 \\
21.6 \\
0 \\
0
\end{array}\right]
$$

$$
\begin{gathered}
x=\left[\begin{array}{c}
\theta_{m} \\
\omega_{m} \\
\theta_{l} \\
\omega_{l}
\end{array}\right], f(x)=\left[\begin{array}{c}
0 \\
0 \\
0 \\
-3.33 \sin \left(x_{3}\right)
\end{array}\right], E=\left[\begin{array}{c}
0.5 \\
1 \\
0 \\
0
\end{array}\right] \\
C=\left[\begin{array}{llll}
1 & 0 & 0 & 0 \\
0 & 1 & 0 & 0
\end{array}\right]
\end{gathered}
$$

The Lipschitz constant of $f(x)$ is $\alpha=0.333 . \omega(t)$ is a Gaussian perturbation bounded by 0.6. By using a nonlinear sector transformation approach [18], a multiple model representation of the above system, which describes exactly the behavior of the original model, is given by (1) with:

$$
\begin{aligned}
A_{1}= & {\left[\begin{array}{cccc}
0 & 1 & 0 & 0 \\
-48.6 & -1.25 & 48.6 & 0 \\
0 & 0 & 0 & 1 \\
19.5 & 0 & -22.83 & 0
\end{array}\right] } \\
A_{2}= & {\left[\begin{array}{cccc}
0 & 1 & 0 & 0 \\
-48.6 & -1.25 & 48.6 & 0 \\
0 & 0 & 0 & 1 \\
19.5 & 0 & -18.77 & 0
\end{array}\right] } \\
B_{1}=B_{2}=B & \left\{\begin{array}{l}
\mu_{1}(z(t))=\frac{z(t)+0.2172}{1.2172} \\
\mu_{2}(z(t))=\frac{1-z(t)}{1.2172}
\end{array}\right.
\end{aligned}
$$

where $z(t)=\frac{\sin \left(x_{3}\right)}{x_{3}}$. The figure 1 (a) presents the states of the original model and those of the multiple model. The weighting functions are illustrated in figure 1 (b).
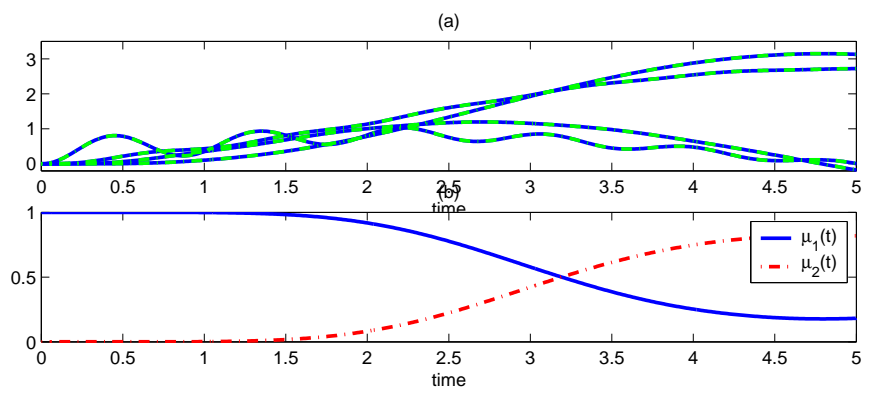

Fig. 1. (a) States of the original and multiple model. (b) Weighting functions

Using the calculation method of Lipschitz constants given in section III, we obtain $\alpha_{1}=\alpha_{2}=16.95$. Due to the fact that $B_{1}=B_{2}=B$ and the property (2) of the weighting functions, we have:

$$
\left.\sum_{i=1}^{r} B_{i}\left[\mu_{i}(x(t))-\mu_{i}(\hat{x}(t))\right)\right] u(t)=0
$$

Then, the term (11) can be reduced as follows:

$$
\Delta(x, \hat{x}, u)=\sum_{i=1}^{r}\left(\bar{A}_{i}\left(\mu_{i}(x(t)) x(t)-\mu_{i}(\hat{x}(t)) \hat{x}(t)\right)\right.
$$


Thus, the constant $\gamma$ is given by:

$$
\gamma=\sum_{i=1}^{r} \bar{\sigma}\left(\bar{A}_{i}\right) \alpha_{i}=0.2027 \alpha_{1}+0.2027 \alpha_{2}=6.8715
$$

Using theorem 3, with $q=0, R=11, \quad a=0.5$, we get:

$$
L=\left[\begin{array}{cc}
9.2540 & 1.8725 \\
-79.1011 & 25.0030 \\
-10.2161 & 5.1087 \\
-36.4449 & 19.1960
\end{array}\right]
$$

The obtained attenuation level is $\xi=0.3084$. Figure 2 displays the simulation results.

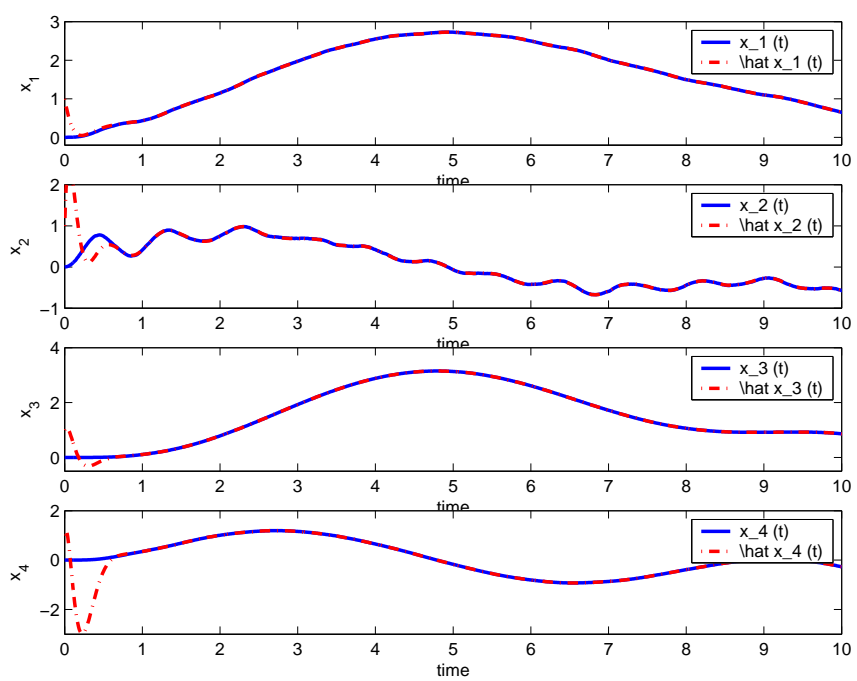

Fig. 2. Actual and estimated states

\section{CONCLUSIONS AND FUTURE WORKS}

In this paper, a method for the state estimation of nonlinear systems is proposed. The Takagi-Sugeno multiple model representation is used. In this work, the most difficult case is considered, namely, where the weighting functions depend on unmeasurable variables (system state). These functions allow to obtain a multiple model which represent exactly the behavior of the initial nonlinear model. The proposed method is less conservative with regard to the value of the Lipschitz constant. Furthermore, finding a common matrix $P$ for all sub-models is difficult or impossible if the number of sub-models is important, what limits the existing methods proposed in the literature. The proposed method reduces the number of LMIs to solve to only one LMI (theorem 1). An extension of the method is proposed to attenuate the effect of bounded exogenous disturbances and the performance of the observer are improved by eigenvalue assignment in a specific LMI region.

\section{REFERENCES}

[1] M. Abbaszadeh and H J. Marquez. A robust observer design method for continuous-time lipschitz nonlinear systems. In Proc. of the Conference on Decision and Control, CDC'2006, San Diego, CA, USA, 2006.

[2] A. Akhenak, M. Chadli, J. Ragot, and D. Maquin. Design of sliding mode unknown input observer for uncertain Takagi-Sugeno model. In 15th Mediterranean Conference on Control and Automation, MED'07, Athens, Greece, 2007.

[3] G. I. Bara, J. Daafouz, F. Kratz, and J. Ragot. Parameter dependent state observer design for affine LPV systems. International Journal of Control, 74(16):1601-1611, November 2001.

[4] P. Bergsten, R. Palm, and D. Driankov. Fuzzy observers. In IEEE International Fuzzy Systems Conference, Melbourne Australia, 2001.

[5] P. Bergsten, R. Palm, and D. Driankov. Observers for Takagi-Sugeno fuzzy systems. IEEE Transactions on Systems, Man, and Cybernetics - Part B: Cybernetics, 32(1):114-121, 2002.

[6] S. Boyd, L. El Ghaoui, E. Feron, and V. Balakrishnan. Linear Matrix Inequalities in System and Control Theory. SIAM ed., 1994.

[7] M. Chadli, D. Maquin, and J. Ragot. Non quadratic stability analysis of Takagi-Sugeno systems. In IEEE Conference on Decision and Control, CDC'2002, Las Vegas, Nevada, USA, 2002.

[8] M. Chilali and P. Gahinet. H-infinity design with pole placement constraints : an LMI approach. IEEE Transactions on Automatic Control, 41(3):358-367, 1996.

[9] J Korbicz, M. Witczak, and V. Puig. LMI-based strategies for designing observers and unknown input observers for non-linear discretetime systems. Bulletin of the Polosh Academy of Sciences, Technical Sciences, 55(1):31-42, 2007.

[10] A. Kruzewski, R. Wang, and T.M. Guerra. Non-quadratic stabilization conditions for a class of uncertain non linear discrete-time T-S fuzzy models: a new approach. IEEE Transactions on Automatic Control, 53(2):606-611, 2008

[11] D.G. Luenberger. An introduction to observers. IEEE Transaction on Automatic Control, 16:596-602, 1971.

[12] R. Palm and P. Bergsten. Sliding mode observers for Takagi-Sugeno fuzzy systems. 9th IEEE International Conference on Fuzzy Systems, FUZZ IEEE 2000, San Antonio, TX, USA, 2000.

[13] R.J. Patton, J. Chen, and C.J. Lopez-Toribio. Fuzzy observers for nonlinear dynamic systems fault diagnosis. In 37th IEEE Conference on Decision and Control, Tampa, Florida USA, 1998.

[14] A.M. Pertew, H.J. Marquez, and Q. Zhao. $\mathrm{H}_{\infty}$ observer design for lipschitz nonlinear systems. IEEE Transactions on Automatic Control, 51:1211-1216, 2006.

[15] S. Raghavan and J. K. Hedrick. Observer design for a class of nonlinear systems. International Journal of Control, 59(2):515-528, 1994.

[16] R. Rajamani. Observers for Lipschitz nonlinear systems. IEEE Transactions on Automatic Control, 43:397-401, March 1998.

[17] T. Takagi and M. Sugeno. Fuzzy identification of systems and its applications to modeling and control. IEEE Transactions on Systems, Man, and Cybernetics, 15:116-132, 1985.

[18] K. Tanaka, T. Ikeda, and H.O. Wang. Fuzzy regulators and fuzzy observers: Relaxed stability conditions and LMI-based designs. IEEE Transactions on Fuzzy Systems, 6(2):250-265, 1998.

[19] F.E. Thau. Observing the state of non-linear dynamic systems. International Journal of Control, 18:471-479, 1973. 